\title{
Atributos textiles de la fibra de alpacas Huacaya blanca y color (Vicugna pacos) de la feria ganadera del sur del Perú
}

\author{
Textile attributes of the fibre of Huacaya white and coloured alpacas (Vicugna \\ pacos) from the livestock fair of south Peru
}

\author{
Jesús E. Quispe Coaquiraa ${ }^{2,3,5}$, Paúl Castillo Paca ${ }^{1}$, William Yana Viveros ${ }^{1}$, \\ Hugo Vilcanqui Mamani ${ }^{2,3}$, Edgar Apaza Zúñiga ${ }^{2,3}$, Diana M. Quispe Roque ${ }^{3,4}$
}

\section{Resumen}

Se caracterizaron las fibras de alpacas Huacaya blances $(n=143)$ y de color $(n=100)$ participantes en la LVIII Feria Ganadera del Sur del Perú (FEGASUR). Las fibras fueron procesadas en el Laboratorio de Fibras del Gobierno Regional Puno. Se describen las frecuencias bajo un modelo de efectos fijos $(\mathrm{p}<0.05)$. Las distribuciones de frecuencias de los atributos de la fibra difieren de la curva normal, en distinto grado y según sea la variable; a excepción del Factor de Confort (FC), presentan un sesgo de cola hacia la derecha; siendo moderada la asimetría del Diámetro Medio (DM) y ligera asimetría del Índice de Curvatura (IC). En tanto, las variables de dispersión, FC y Largo de mecha (LM) presentaron curvas muy asimétricas. Las fibras blancas exhiben mayor finura que las de color $(\mathrm{p}<0.05)$, sin diferencias para el factor sexo. La Desviación estándar del diámetro medio [DS(DM)] y el Coeficiente de variación del diámetro medio [CV(DM)] de las fibras blancas tuvieron menor dispersión $(\mathrm{p}<0.05)$, en tanto que el FC y el IC presentaron

\footnotetext{
${ }^{1}$ Proyecto Especial de Camélidos Sudamericanos, PECSA. Gobierno Regional Puno, Perú

${ }^{2}$ Universidad Nacional del Altiplano, Puno, Perú

${ }^{3}$ Instituto de Investigación y Promoción de Camélidos Sudamericanos, UNA Puno, Perú

${ }^{4}$ Universidad Nacional Micaela Bastidas, Apurímac, Perú

${ }^{5}$ E-mail: jesusquispecoaquira@gmail.com
}

Recibido: 15 de noviembre de 2020

Aceptado para publicación: 29 de mayo de 2021

Publicado: 24 de agosto de 2021

CLos autores. Este artículo es publicado por la Rev Inv Vet Perú de la Facultad de Medicina Veterinaria, Universidad Nacional Mayor de San Marcos. Este es un artículo de acceso abierto, distribuido bajo los términos de la licencia Creative Commons Atribución 4.0 Internacional (CC BY 4.0) [https:// creativecommons.org/licenses/by/4.0/deed.es] que permite el uso, distribución y reproducción en cualquier medio, siempre que la obra original sea debidamente citada de su fuente original 
mejores valores $(\mathrm{p}<0.05)$. El LM no mostró diferencias entre fibras blancas y de color. Los atributos, en función a la categoría de las alpacas, se relacionan con la edad, a excepción del CV(DM). Se concluye que las alpacas Huacaya de plantel, blanca o de color, difieren entre sí y son peculiares respecto a los reportes que no tipifican el tipo de rebaño.

Palabras clave: dispersión, curvatura, camélidos, altiplano, Puno

\section{AbSTRACT}

The fibres of white $(n=143)$ and coloured $(n=100)$ Huacaya alpacas participating in the LVIII Livestock Fair of Southern Peru (FEGASUR) were characterized. The fibres were processed in the Fibre Laboratory of the Puno Regional Government. The frequencies were described under a fixed effects model $(p<0.05)$. The frequency distributions of the fibre attributes differ from the normal curve to a different degree and depending on the variable, except for the Comfort Factor (CF), they show a tail bias to the right; being moderate the asymmetry of the Mean Diameter (MD) and slight asymmetry of the Index of Curvature (IC). Meanwhile, the dispersion variables, CF and Fibre length (LM) presented very asymmetric curves. White fibres show greater fineness than coloured fibres $(\mathrm{p}<0.05)$, without differences for the sex factor. The standard deviation of the mean diameter [SD $(\mathrm{DM})]$ and the Coefficient of variation of the mean diameter [CV (DM)] of the white fibres had lower dispersion $(p<0.05)$, while the CF and IC presented better values $(p<0.05)$. The LM did not show differences between white and coloured fibres. The attributes, depending on the category of the alpacas, are related to age, except for $\mathrm{CV}(\mathrm{DM})$. It is concluded that the Huacaya alpacas of the breeding stock, white or coloured, differ from each other and are peculiar with respect to the reports that do not typify the type of herd.

Key words: dispersion, curvature, camelids, highland plateau, Puno

\section{INTRODUCCIÓN}

El 97\% de la población de alpacas se encuentra en los Andes peruanos, por encima de los 3500 msnm, y bajo el cuidado de pequeños y medianos criadores, junto a una mínima proporción de especies alóctonas crioIlas (ovinosy vacunos) (Quispe et al., 2016). En el Perú se reporta 3.6 millones de alpacas de dos razas definidas: Huacaya (79\%) y Suri (12\%) (CENAGRO, 2013; Quispe et al., 2016), siendo la región Puno la que concentra la mayor población de alpacas del país (39.6\%) (Quispe et al., 2016).
La alpaca Huacaya presenta un fenotipo distintivo con cabeza pequeña, cuello largo y fuerte y orejas triangulares, a la vez que un vellón de mechas cortas, más rizadas y compactas, que le confieren una corpulencia y fortaleza particular (Bustinza, 2001; Quispe et al., 2016). La alpaca produce una fibra especial que la industria textil la clasifica como fibra de lujo, siendo apreciada por su suavidad, peso ligero, gama de colores y buena resistencia (Wang et al., 2003). De la misma manera, los consumidores las eligen por sus cualidades térmicas, resistencia, impermeabilidad y no ser inflamables (Atav y Turkmen, 2006). 
En la crianza de alpacas se mantienen dos grupos: plantel y majada general, siendo primero orientado a la producción comercial de reproductores, usualmente registrados en la Asociación de Criadores de Alpacas, y el segundo se dirige a la producción de fibra y carne. Dichos sistemas difieren sustancialmente en las prácticas de mejora genética, manejo, alimentación y prácticas de bienestar y salud animal (Quispe et al., 2016).

En la década del 70 se iniciaron las exposiciones y concursos de alpacas blancas en la zona del altiplano, motivado por las exigencias de la industria textil por fibra blanca, dada sus ventajas en el teñido industrial. Esto originó el descarte masivo e indiscriminado de las alpacas de color de los rebaños. No obstante, a raíz de la casi extinción de las alpacas color se inició la participación de alpacas con vellones de colores enteros desde inicios de presente milenio en estas exposiciones, lo que propició la formación de Bancos de Germoplasma para evitar la pérdida de dicha biodiversidad (Bustinza, 2001). En las alpacas existe una amplia gama de colores de vellón que varían desde el blanco hasta el negro, sobresaliendo en la actualidad los vellones simples (leonado claro [LF], café y negro, en sus distintas tonalidades). Sin embargo, existe una menor frecuencia de colores compuestos binarios (gris, roano), aunque no se acepta para propósitos de concurso a las alpacas con vellones compuestos múltiples o moteados (Quispe et al., 2016). En sí, para los criadores de alpacas de plantel, las ferias constituyen el escenario de exposición y concurso de alpacas de la mayor calidad genética.

La mayor parte de los estudios sobre atributos morfológicos y textiles de la fibra no han considerado el tipo de rebaño (plantel o majada). En virtud de ello, el presente estudio tuvo como objetivo describir las frecuencias de los atributos de la fibra; determinar las características tecnológicas y el grado de asociación entre atributos de la fibra de alpacas Huacaya admitidas en la LVIII Feria Ganadera del Sur del Perú (FEGASUR
2019), teniendo en cuenta factores como el sexo, patrón de color del vellón y la categoría de juzgamiento.

\section{Materiales y Métodos}

El estudio se llevó a cabo en el campo ferial de la LVIII Feria Ganadera, Artesanal y Agroindustrial del Sur (FEGASUR), ubicado en la ciudad de Juliaca, provincia de San Román de la Región Puno, Perú. La zona se encuentra a una altitud de $3833 \mathrm{msnm}$. Se utilizaron 243 muestras de vellón de alpacas Huacaya (143 muestras de alpacas blancas y 100 de alpacas de color) que fueron admitidas a la exposición y concurso en la LVIII FEGASUR 2019 (Figura 1). Las alpacas provenían de distintos núcleos de crianza de reproductores de alpacas Huacaya de la Región Puno. Estos centros de crianza se encuentran en las dos zonas agroecológicas que circundan el Altiplano peruano: puna húmeda (Carabaya, Huancané, Melgar, San Antonio de Putina) y puna seca (El Collao, Lampa y San Román). De manera general, el 82\% de las alpacas Huacaya pertenecen a rebaños de plantel de la puna húmeda.

\section{Análisis de Fibra}

Las muestras de fibra fueron tomadas del costillar medio de la alpaca según la recomendación de Aylan-Parker y McGregor (2002) y colocadas en bolsas de polietileno. Para el estudio, se consideraron los factores sexo (macho y hembra), patrón de color del vellón (blanco y color) y categorías de juzgamiento (A1, A2, B, C y D), siendo A1: tui menor, A2: tui mayor, B: dos dientes, $\mathrm{C}$ : cuatro dientes, y D: seis dientes.

Los análisis de las características textiles se llevaron a cabo en el Laboratorio de Fibras del Proyecto Especial Camélidos Sudamericanos (PECSA) del Gobierno Regional Puno, utilizando un equipo OFDA 2000, instrumento diseñado y propuesto para la medición de diferentes características en muestra 

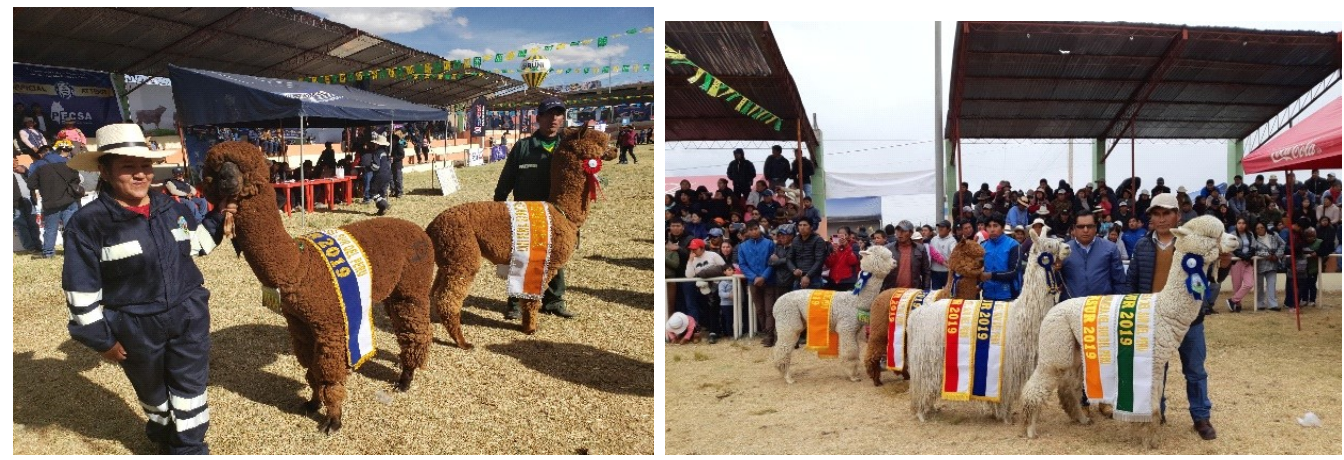

Figura 1. Alpacas Huacaya blanca y de color, campeones de la FEGASUR 2019

de fibra grasienta, con rapidez y precisión (IWG, 2000) y reconocido por la International Wool Textile Organisation (IWTO).

Como variables respuesta se consideraron los siguientes atributos: diámetro medio de la fibra, desviación estándar y coeficiente de variabilidad del diámetro de fibra, factor de confort, índice de curvatura y largo de la mecha. Para la descripción de las variables respuesta se utilizó las distribuciones de frecuencias y medidas de tendencia central y dispersión. Para el análisis de los atributos tecnológicos de la fibra se planteó un arreglo factorial de $2 \times 2 \times 5$ (sexo x patrón de color $x$ categoría de juzgamiento) conducido en un Diseño Completo al Azar, mientras que la comparación de medias fue a través de la prueba de significancia de Duncan $(\mathrm{p}<0.05)$. Asimismo, el grado de asociación entre los atributos se hizo mediante el coeficiente de correlación de Pearson.

\section{Resultados y Discusión}

\section{Frecuencias de los Atributos de la Fibra}

El Cuadro 1 y la Figura 2 presentan los principales estadísticos y distribuciones de frecuencias de los atributos de la fibra de alpacas blanca y de color, que participaron en la LVIII Feria Ganadera, Artesanal y Agroindustrial del Sur (FEGASUR).

En las alpacas blancas, la distribución de frecuencias del DM e IC muestran una distribución platicúrtica, en tanto que los demás atributos muestran una distribución leptocúrtica; es decir, en el primero los datos se encuentran más extensamente distribuidos alrededor de la media; y en el segundo los datos se encuentran condensados hacia la media, lo cual le confiere el típico apuntalamiento particular. El coeficiente de asimetría de los atributos, a excepción del FC, presentan un sesgo de cola hacia la derecha; y con base al índice curtosis de Pearson. El DM presenta una moderada asimetría y el IC una ligera asimetría; mientras que las variables DS(DM), CV(DM), FC y LM presentan curvas muy asimétricas (Martínez, 1999; Toma y Rubio, 2020).

En las alpacas de color, los atributos DM, DS(DM) y CV(DM) muestran una distribución platicúrtica, y las demás presentan una distribución leptocúrtica. Con base al índice de curtosis, el DM, DS(DM) e IC presentan una asimetría moderada, el CV(DM) muestra ligera asimetría, y el FC y LM una asimetría moderada. 
Cuadro 1. Principales estadísticos de los atributos textiles de la fibra de alpaca Huacaya, de animales participantes de la LVIII Feria Ganadera, Artesanal y Agroindustrial del Sur (FEGASUR). Puno, Perú, 2019

\begin{tabular}{lcccccc}
\hline Patrón de color & DM & DS(DM) & CV(DM) & FC & IC & LM \\
\hline Blanca & & & & & & \\
$\quad$ Media & 19.599 & 4.736 & 24.251 & 96.544 & 57.518 & 70.810 \\
Mediana & 19.250 & 4.650 & 23.800 & 97.800 & 57.900 & 60.000 \\
Moda & 18.400 & 4.100 & 23.000 & 98.800 & 59.300 & 60.000 \\
Desv. estándar & 2.486 & 0.715 & 2.849 & 3.408 & 8.469 & 28.017 \\
Curtosis & -0.455 & 0.769 & 2.317 & 6.597 & -0.006 & 0.638 \\
Coef. asimetría & 0.443 & 0.802 & 0.996 & -2.422 & 0.196 & 1.110 \\
Color & & & & & & \\
$\quad$ Media & 21.84 & 5.53 & 25.40 & 91.38 & 50.47 & 68.54 \\
$\quad$ Mediana & 21.500 & 5.300 & 25.300 & 94.900 & 50.300 & 60.000 \\
$\quad$ Moda & 20.300 & 5.600 & 25.900 & 98.700 & 51.500 & 50.000 \\
$\quad$ Desv. estándar & 3.486 & 1.028 & 3.100 & 9.179 & 9.320 & 26.547 \\
Curtosis & -0.156 & -0.179 & -0.255 & 3.909 & 1.249 & 1.484 \\
Coef. asimetría & 0.365 & 0.593 & 0.285 & -1.943 & -0.496 & 1.203 \\
\hline DM = Diámetro Medio, DS(DM) & $=$ Desviación estándar del diámetro medio, CV(DM) = \\
Coeficiente de variación del diámetro medio, FC= Factor de confort, IC = Índice de curvatura, \\
LM = Longitud de mecha
\end{tabular}

Cuadro 2. Características textiles de la fibra de alpacas Huacaya (Promedio \pm EE) participantes de la LVIII Feria Ganadera, Artesanal y Agroindustrial del Sur (FEGASUR). Puno, Perú, por color y sexo. 2019

\begin{tabular}{lcccc}
\hline \multirow{2}{*}{ Variable } & \multicolumn{2}{c}{ Blanco } & \multicolumn{2}{c}{ Color } \\
\cline { 2 - 5 } & Macho & Hembra & Macho & Hembra \\
\hline Animales (n) & 77 & 66 & 57 & 43 \\
DM, $\mu \mathrm{m}$ & $19.59 \pm 0.27^{\mathrm{b}}$ & $19.27 \pm 0.29^{\mathrm{b}}$ & $21.76 \pm 0.50^{\mathrm{a}}$ & $21.43 \pm 0.56^{\mathrm{a}}$ \\
DS(DM), $\mu \mathrm{m}$ & $4.73 \pm 0.08^{\mathrm{b}}$ & $4.71 \pm 0.09^{\mathrm{b}}$ & $5.64 \pm 0.14^{\mathrm{a}}$ & $5.28 \pm 0.16^{\mathrm{a}}$ \\
CV $(\mathrm{DM}), \%$ & $24.19 \pm 0.31^{\mathrm{b}}$ & $24.65 \pm 0.34^{\mathrm{ab}}$ & $25.99 \pm 0.43^{\mathrm{a}}$ & $24.78 \pm 0.49^{\mathrm{ab}}$ \\
FC, \% & $96.34 \pm 0.39^{\mathrm{a}}$ & $97.01 \pm 0.43^{\mathrm{a}}$ & $91.44 \pm 1.29^{\mathrm{b}}$ & $92.50 \pm 1.45^{\mathrm{b}}$ \\
IC, $/ \mathrm{mm}$ & $57.12 \pm 1.03^{\mathrm{a}}$ & $58.02 \pm 1.12^{\mathrm{a}}$ & $49.95 \pm 1.36^{\mathrm{b}}$ & $50.99 \pm 1.52^{\mathrm{b}}$ \\
LM, mm & $67.67 \pm 3.26^{\mathrm{a}}$ & $69.36 \pm 3.55^{\mathrm{a}}$ & $63.77 \pm 3.72^{\mathrm{a}}$ & $67.60 \pm 4.18^{\mathrm{a}}$ \\
\hline
\end{tabular}

Superíndices diferentes dentro de filas indican diferencias significativas entre medias $(\mathrm{p}<0.05)$ $\mathrm{DM}=$ Diámetro Medio, $\mathrm{DS}(\mathrm{DM})=$ Desviación estándar del diámetro medio, $\mathrm{CV}(\mathrm{DM})=$ Coeficiente de variación del diámetro medio, $\mathrm{FC}=$ Factor de confort, $\mathrm{IC}=$ Índice de curvatura, $\mathrm{LM}=$ Longitud de mecha 


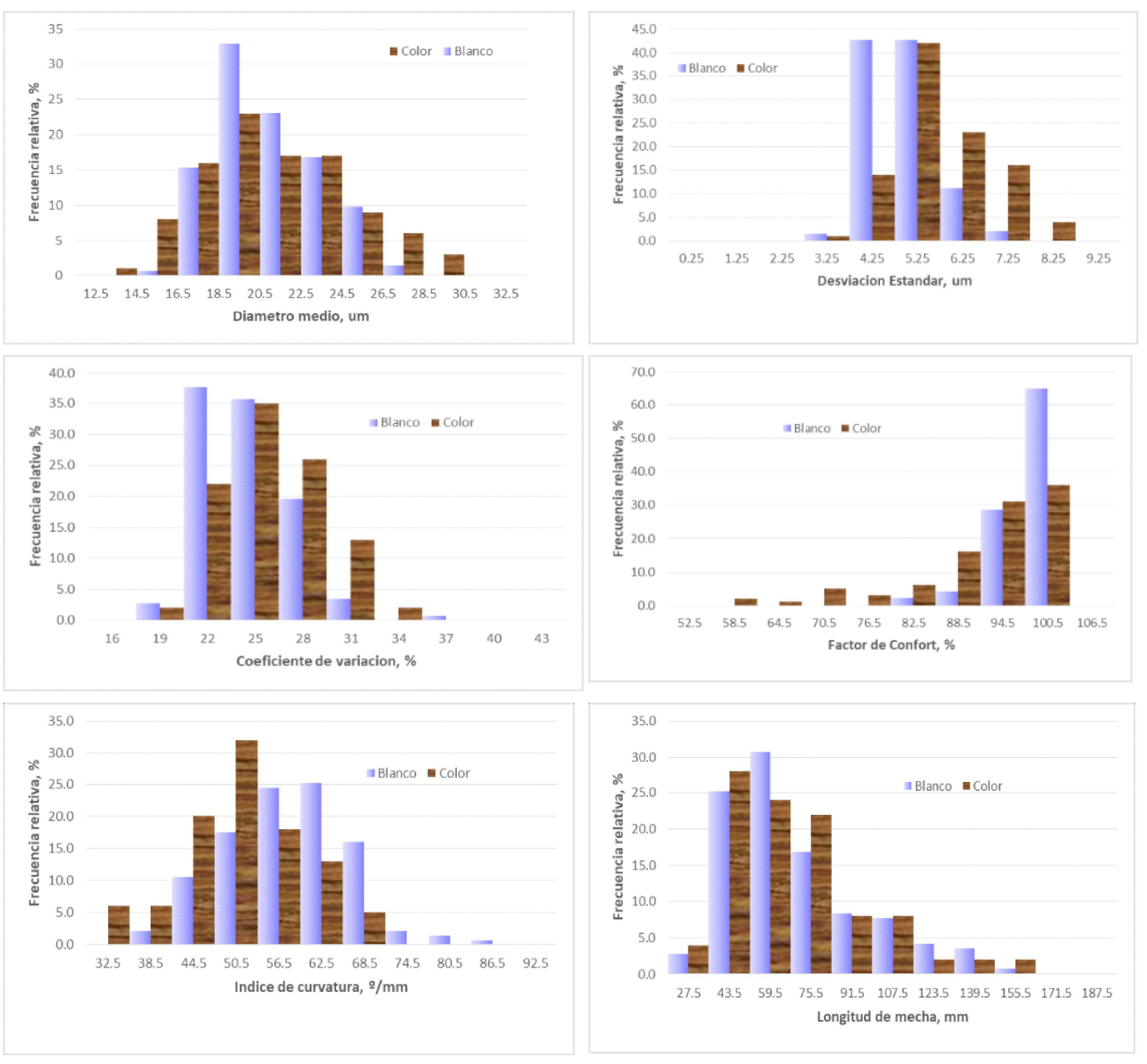

Figura 2. Distribución de frecuencias relativas de las características tecnológicas de la fibra de alpacas Huacaya participantes de la LVIII Feria Ganadera, Artesanal y Agroindustrial del Sur (FEGASUR). Puno, Perú, 2019

En consecuencia, se puede inferir que i) las fibras blanca y de color de las alpacas Huacaya bajo evaluación difieren de la curva normal en distinto grado y según sea la variable, siendo menos acentuada en las alpacas de color; ii) las peculiares distribuciones de las fibras de alpacas están relacionadas con la intensidad de selección aplicada y el tiempo de formación de los rebaños de cría; iii) probablemente, el criador ha elegido la selección de alpacas con vellón blanco y tiempo después orientó hacia la producción de fibra fina. De esta manera, se puede indicar que se aplica una selección dirigida en los rebaños de plantel, lo que ha inducido a que la distribución del DM sea más alta hacia el extremo de fibras finas y sesgada a la cola derecha. Esto se observa también con las variables $\mathrm{DS}(\mathrm{DM}), \mathrm{CV}(\mathrm{DM})$, mientras que el FC muestra un comportamiento opuesto. En síntesis, la distribución peculiar del DM de la fibra blanca corrobora que no solo está 
Cuadro 3. Características textiles de la fibra de alpacas Huacaya (promedio \pm EE) participantes de la LVIII Feria Ganadera, Artesanal y Agroindustrial del Sur (FEGASUR). Puno, Perú, por color y categoría de juzgamiento. 2019

\begin{tabular}{|c|c|c|c|c|c|c|c|}
\hline & $\mathrm{n}$ & $\mathrm{DM}, \mu \mathrm{m}$ & $\mathrm{DS}(\mathrm{DM}), \mu \mathrm{m}$ & $\mathrm{CV}(\mathrm{DM}), \%$ & $\mathrm{FC}, \%$ & $\mathrm{IC}, \% / \mathrm{mm}$ & $\mathrm{LM}, \mathrm{mm}$ \\
\hline \multicolumn{8}{|c|}{ Blanco } \\
\hline A1 & 17 & $17.23 \pm 0.55^{\mathrm{d}}$ & $4.51 \pm 0.17^{\mathrm{c}}$ & $26.28 \pm 0.64^{\mathrm{a}}$ & $98.19 \pm 0.79^{\mathrm{a}}$ & $54.82 \pm 2.08^{\mathrm{abc}}$ & $49.41 \pm 6.62^{b}$ \\
\hline A2 & 44 & $19.08 \pm 0.34^{\mathrm{cd}}$ & $4.62 \pm 0.11^{\mathrm{c}}$ & $24.25 \pm 0.40^{\mathrm{ab}}$ & $97.18 \pm 0.49^{\mathrm{ab}}$ & $56.94 \pm 1.29 \mathrm{abc}$ & $72.84 \pm 4.11^{\mathrm{ab}}$ \\
\hline B & 29 & $19.56 \pm 0.42^{\mathrm{bcd}}$ & $4.80 \pm 0.13^{\mathrm{bc}}$ & $24.55 \pm 0.49^{\mathrm{ab}}$ & $96.87 \pm 0.61^{\mathrm{ab}}$ & $59.26 \pm 1.60^{\mathrm{a}}$ & $72.16 \pm 5.09 \mathrm{ab}$ \\
\hline $\mathrm{C}$ & 22 & $20.4 \pm 0.48^{a b c}$ & $4.90 \pm 0.15^{\mathrm{abc}}$ & $24.06 \pm 0.56^{\mathrm{ab}}$ & $95.88 \pm 0.70^{\mathrm{abc}}$ & $58.19 \pm 1.84^{\mathrm{a}}$ & $68.08 \pm 5.83^{a b}$ \\
\hline $\mathrm{D}$ & 31 & $20.89 \pm 0.41^{\mathrm{abc}}$ & $4.79 \pm 0.13^{\mathrm{bc}}$ & $22.96 \pm 0.48^{b}$ & $95.26 \pm 0.60^{\mathrm{abcd}}$ & $58.64 \pm 1.56^{\mathrm{a}}$ & $80.09 \pm 4.96^{\mathrm{a}}$ \\
\hline \multicolumn{8}{|c|}{ Color } \\
\hline A1 & 11 & $20.00 \pm 1.06^{\mathrm{bc}}$ & $4.93 \pm 0.30^{\mathrm{abc}}$ & $25.00 \pm 0.92^{\mathrm{ab}}$ & $95.95 \pm 2.73^{\mathrm{abc}}$ & $47.59 \pm 2.87^{c}$ & $49.83 \pm 7.87^{\mathrm{b}}$ \\
\hline $\mathrm{A} 2$ & 33 & $21.48 \pm 0.61^{\mathrm{abc}}$ & $5.54 \pm 0.18^{\mathrm{ab}}$ & $25.79 \pm 0.53^{\mathrm{a}}$ & $91.42 \pm 1.59^{\mathrm{bcd}}$ & $48.71 \pm 1.67^{\mathrm{bc}}$ & $74.54 \pm 4.58^{a b}$ \\
\hline B & 17 & $21.33 \pm 0.85^{\mathrm{abc}}$ & $5.57 \pm 0.24^{\mathrm{ab}}$ & $26.18 \pm 0.74^{\mathrm{a}}$ & $93.13 \pm 2.19^{\mathrm{abcd}}$ & $52.32 \pm 2.30^{\mathrm{abc}}$ & $68.82 \pm 6.31^{\mathrm{ab}}$ \\
\hline $\mathrm{C}$ & 17 & $22.21 \pm 0.85^{\mathrm{ab}}$ & $5.56 \pm 0.24^{\mathrm{ab}}$ & $25.04 \pm 0.74^{\mathrm{ab}}$ & $90.59 \pm 2.19^{\mathrm{cd}}$ & $52.03 \pm 2.30^{\mathrm{abc}}$ & $67.33 \pm 6.31^{\mathrm{ab}}$ \\
\hline $\mathrm{D}$ & 22 & $22.95 \pm 0.77^{\mathrm{a}}$ & $5.68 \pm 0.22^{\mathrm{a}}$ & $24.90 \pm 0.67^{\mathrm{ab}}$ & $88.76 \pm 2.00^{\mathrm{d}}$ & $51.71 \pm 2.10^{\mathrm{abc}}$ & $67.90 \pm 5.76^{\mathrm{ab}}$ \\
\hline
\end{tabular}

Superíndices diferentes dentro de columnas indican diferencias significativas entre medias $(p<0.05)$ $\mathrm{DM}=$ Diámetro Medio, DS(DM) = Desviación estándar del diámetro medio, $\mathrm{CV}(\mathrm{DM})=$ Coeficiente de variación del diámetro medio, FC = Factor de confort, IC = Índice de curvatura, LM = Longitud de mecha

relacionado con la intensidad de selección aplicada en los rebaños de cría, sino también con el tiempo de formación de estos.

\section{Características Textiles de la Fibra}

El juzgamiento de las alpacas blancas y de color se realizó por separado conforme estipula el Reglamento Ferial y la Ley de Registros Genealógicos de Camélidos Sudamericanos del Perú (RGALLP, 2011). Los promedios de las características textiles de la fibra por color y sexo se presentan en el Cuadro 2, y por color y categoría de juzgamiento en el Cuadro 3, e incluyen las cinco categorías de juzgamiento

\section{Diámetro Medio de la Fibra}

Las alpacas blancas, macho y hembra, exhiben mayor finura $(19.59$ y $19.27 \mu \mathrm{m}$, respectivamente) que las alpacas de color (21.76 y $21.43 \mu \mathrm{m}$, respectivamente $)(\mathrm{p}<0.05)$; $\sin$ embargo, no hubo diferencias para el factor sexo dentro de cada color. La diferencia de medias implica poco más de dos micras, y en términos relativos representa el $11 \%$ a favor de la fibra blanca. Es importante precisa que el promedio de finura no excede de $20 \mu \mathrm{m}$ en las alpacas blancas y de $22 \mu \mathrm{m}$ en las de color, lo que indica la calidad genética alcanzada en los rebaños de cría presentes en la feria. La expresión de la mayor finura en las alpacas blancas es atribuible al apareamiento selectivo y sistemático aplicado por los criadores para atender las exigencias del mercado textil; y cumplir con los estándares raciales fijados en el Reglamento de Registros Genealógicos de Alpacas y Llamas del Perú (RGALLP, 2011).

Según se puede observar en el Cuadro 3 , las alpacas blancas de la categoría A1 presentaron la mayor finura $(17.23 \mu \mathrm{m})$, en tanto que el mayor DM correspondió a la categoría D $(20.89 \mu)$. En el caso de las alpacas 
de color, la categoría D expresó el mayor grosor de fibra $(22.95 \mu \mathrm{m})$ y la mayor finura correspondió a la categoría A1 $(20 \mu \mathrm{m})$. En términos absolutos, la diferencia entre medias de categorías extremas (A1 y D) fue de $3.66 \mu \mathrm{m}$ en las alpacas blancas y de $2.95 \mu \mathrm{m}$ en las de color, y que en términos relativos significa 21 y $14 \%$, respectivamente.

En la caracterización del DM de la fibra de alpacas blancas, Vásquez et al. (2015), Machaca et al. (2017) y Meza (2018) señalan diferencias significativas para el factor sexo $(p<0.05)$. Por otro lado, medias similares fueron reportados por Vásquez et al. (2015) en Apurímac (19.6 y $20.1 \mu \mathrm{m}$ para machos y hembras, respectivamente) y Cutiri (2019) en Cusco (19.82 y 20.58 ìm para machos y hembras, respectivamente). Los reportes de Gil (2017) y Quispe et al. (2020) en la región Puno; Siña (2012) al sur del país y Barrionuevo (2019), Contreras (2010), Castillo y Zacarías (2014), Arango (2016), Machaca et al. (2017) y Meza (2018) hacia el norte de la región señalan DM superiores al presente estudio.

Al comparar los resultados encontrados en las alpacas blancas, las medias de la categoría A 1 fueron similares a las reportadas por Cutiri (2019) (17.33 $\mu \mathrm{m}$, en dientes de leche), pero menores a las obtenidas por otros autores que señalan valores superiores a $19.5 \mu \mathrm{m}$ (Contreras, 2010; Siña, 2012; Arango, 2016; Gil, 2017; Quispe et al., 2020). En el caso de la categoría D, Vásquez et al. (2015) y Cutiri (2019) señalan medias similares a las del presente estudio (20.89 ìm), pero Gil (2017) refiere valores superiores a $26 \mu \mathrm{m}$ en hembras de ocho años.

Del conjunto de referencias se deduce que i) es evidente las diferencias de finura entre alpacas blanca y de color; las cuales se pueden atribuir al tiempo de formación de los planteles; ii) que las alpacas en los centros de recría son seleccionadas para la producción de fibra fina, conservando aspectos mí- nimos de la conformación corporal; y iii) existe una amplia diversidad feno-genotípica del DM de alpacas blancas, que varían en función a sistemas de crianza, localización geográfica, estrategias de manejo, entre otros.

No se encontraron referencias relacionadas a la finura de la fibra de alpacas de color, con excepción de Barrionuevo (2019) quién reporta DM para animales café ( 24.42 $\mu \mathrm{m})$ y negro $(25.61 \mu \mathrm{m})$, indicando además que hubo diferencias a favor de la fibra blanca $(p<0.05)$. Al parecer el color del vellón, genéticamente de naturaleza mendeliana, no tiene efectos en la expresión de la finura; empero esta característica de importancia económica, por su naturaleza cuantitativa, responde a la presión de selectiva aplicada en los rebaños de plantel.

\section{Desviación Estándar del Diámetro Medio}

La DS(DM) de las alpacas blancas, macho y hembra, muestran menores valores $(4.73$ y $4.71 \mu \mathrm{m})$ respecto a las de color $(5.64$ y $5.28 \mu \mathrm{m}$, respectivamente) $(\mathrm{p}<0.05)$; es decir, las fibras blancas tienen menor dispersión alrededor de la media del DM. Las diferencias entre medias de fibras blanca y color, en términos absolutos significan 0.91 y $0.47 \mu \mathrm{m}$, para machos y hembras, respectivamente; siendo 19 y $10 \%$ en términos relativos, respectivamente. En suma, las medias del DS(DM) de alpacas blancas no exceden del $5 \%$ y las de color del $6 \%$.

La DS también se relaciona con la edad de la alpaca, pues valores bajos de dispersión correspondieron a los de menor edad. Las diferencias absolutas entre categorías extremas fueron 0.28 y 0.75 ìm en blanca y de color, respectivamente, lo que en términos relativos representaron el 6 y $15 \%$, respectivamente. Es decir, la fibra blanca de las categorías A1 y A2 tienen la menor dispersión; y las de color de la categoría D se caracterizan por su mayor dispersión. En la literatura revisada, en alpacas blancas de la sierra cen- 
tral del país, Castillo y Zacarías (2014) y Arango (2016) reportan DS(DM) mayor heterogeneidad de finura respecto a las alpacas de plantel.

En cuanto a la categoría etaria, los resultados coinciden con los reportes de Castillo y Zacarías (2014) y Arango (2016), quienes señalan que las alpacas juveniles presen$\tan$ mayor homogeneidad (4.99 y $4.19 \mu \mathrm{m})$ que las de mayor edad (5.73 y $5.44 \mu \mathrm{m})$. Cabe indicar que a menor DS(DM) más uniformemente dimensionada estarán las fibras, lo cual es deseable, para propósitos del procesamiento textil (Holt y Scott, 2006).

\section{Coeficiente de Variación del Diámetro Medio}

Las alpacas macho blanco exhiben menor variación del CV(DM) $(24.19 \%)$, en tanto que las de color tienen mayor variación (25.99\%). De otra parte, las hembras blancas $(24.65 \%)$ y de color $(24.78 \%)$ exhiben niveles de dispersión intermedios. En términos absolutos implican diferencias del 3.32 y $1.28 \%$ para machos y hembras, y en términos relativos representan el 14 y $5 \%$, respectivamente.

Gil (2017) en alpacas blancas hembra del CE La Raya, Puno, y Barrionuevo (2019) en comunidades de Ocongate, Cusco, reportaron valores similares al presente estudio; en tanto que Castillo y Zacarías (2014) en Huancavelica, Vásquez et al. (2015) en la zona altoandina de Apurímac y Cutiri (2019) en Ocongate, Cusco, reportaron valores menores de $\mathrm{CV}(\mathrm{DM})$.

El CV(DM) en alpacas blancas varía entre 22 y $26 \%$ y en color entre 24 y $27 \%$, según la edad, siendo un rango de variación menor al obtenido en los estudios anteriormente mencionados. En este sentido, se puede indicar que altos valores de CV(DM) se relacionan con rebaños sin mayor presión de selección como ocurre en los rebaños de majada. Asimismo, se corrobora que, en el tiempo, el apareamiento de alpacas de «lo mejor con lo mejor» aplicado en los rebaños de cría viene dando resultados de mayor finura y uniformidad de fibra.

En alpacas Huacaya se afirma que un $24 \%$ del CV sea el límite para el rendimiento textil acorde a su diámetro y que está asociada al rendimiento del hilado o diámetro ajustado a la hilatura (Quispe, 2010). Al respecto, Cottle (2010) precisa que vellones con CV(DM) bajos son evidencia de una mayor uniformidad de finura de fibras individuales dentro del vellón. En tal virtud, se reconoce dos fuentes de variación: una referida a la variación dentro de la mecha, que se estima sea responsable del $80 \%$ de la variación; es decir, al CV(DM), y la otra referida a la variación a lo largo de la fibra (perfil de la fibra), responsable del $20 \%$ de la variación (Botha y Hunter, 2010).

\section{Factor de Confort}

Las alpacas blancas, machos y hembras, presentan valores superiores $(96.34 \mathrm{y}$ $97.01 \%$, respectivamente) que las de color (91.44 y $92.50 \%$, respectivamente $)(\mathrm{p}<0.05)$., lo que indica que las fibras blancas brindan mayor comodidad; empero, dentro de cada color no hubo diferencias entre sexos. Las diferencias relativas para el factor sexo fluctúan entre 5 y $6 \%$.

El FC está relacionado con la edad. Alpacas de la categoría A1 exhiben altos índices de confort, disminuyendo conforme avanza la edad. Las alpacas blancas de la categoría A1 tienen el más alto valor de confort $(98.19 \%)$ y las alpacas de color de la categoría D $(88.76 \%)$ se caracterizan por su menor factor de comodidad. Es interesante notar que a excepción de la categoría A1 color, las demás categorías de alpacas de color no reúnen las condiciones mínimas de confort que demanda la industria textil; en cambio, todas las categorías de las alpacas blancas reúnen el nivel de confort mínimo (95\%). 
Las medias del FC de las alpacas blancas del estudio fueron similares al $96 \%$ registrado por Vásquez et al. (2015) en Apurímac y al 95.4\% por Barrionuevo (2019) en Cusco; mientras que otros autores reportan valores inferiores (Cutiri, 2014; Arango, 2016; Gil, 2017; Machaca et al., 2017; Meza, 2018). Por otro lado, solo Vásquez et al. (2015) y Machaca et al. (2017) refieren diferencias significativas para el factor sexo. Los resultados corroboran que el FC, definido como el porcentaje de fibras menores a 30 $\mu \mathrm{m}$ (McColl et al., 2004), está directamente relacionado con la mayor finura las fibras que brindan mayor comodidad (McGregor y Butler, 2004); aspecto de gran importancia para la industria textil, pues los extremos de las fibras mayores a 30 ìm causan irritación mecánica en la piel, debido a su menor flexibilidad.

\section{Índice de Curvatura}

El IC de las alpacas blancas (57.12 y $58.02 \% \mathrm{~mm}$ ) presenta mayores valores que las alpacas de color $\left(49.95\right.$ y $\left.50.99^{\circ} / \mathrm{mm}\right)$ en machos y hembras ( $<<0.05$; Cuadro 2). Las diferencias entre alpacas blanca y de color en términos absolutos fluctúan entre 7 y $8 \% \mathrm{~mm}$; y que en valores relativos están entre $14 \mathrm{y}$ $16 \%$ a favor de la fibra blanca.

El IC guarda alguna relación directa con la edad, pues alpacas de la categoría A1 exhiben los índices bajos (Cuadro 3). Las diferencias absolutas entre clases extremas fueron 3.82 y $4.22 \%$ mm, en alpacas blancas y color (7 y $8 \%$, respectivamente). Los resultados muestran que las alpacas blancas de la categoría $\mathrm{D}$ tenían el IC más alto.

Los IC hallados por Vásquez et al. (2015), Machaca et al. (2017), Gil (2017), Barrionuevo (2019) y Quispe et al. (2020) son menores a $39 \% \mathrm{~mm}$, en tanto que no encontraron una tendencia definida con la edad del animal. En el presente estudio resalta el IC de las alpacas blancas de plantel $(58 \% \mathrm{~mm})$ respecto a las alpacas de color $(50 \% \mathrm{~mm})$.
El IC de la fibra mide el ángulo en el rizado de la fibra, o sea tiene estrecha relación con la frecuencia de rizos. Así, a mayor valor del IC habrá mayor frecuencia de rizos; lo que corrobora el alto grado de ondulación o rizado de las fibras que se observa comúnmente en las alpacas de plantel (Bustinza, 2001; Quispe et al., 2016). Por lo general, el IC es una característica adicional utilizada para describir la propiedad espacial de una masa de fibras. Incluso los fabricantes de fibras sintéticas introducen rizos a sus fibras y filamentos para mejorar la densidad de sus productos textiles (Fish et al., 1999).

Holt (2006) enfatiza que si el IC es menor a $20 \% \mathrm{~mm}$ se la califica como curvatura baja, entre 40 y $50 \% \mathrm{~mm}$ se considera de curvatura media, y si excede de $50^{\circ} / \mathrm{mm}$ la califica como curvatura alta. Con base de ello, se puede afirmar que las fibras de las alpacas blancas del presente estudio presentan curvatura alta y las de color tienen curvatura media.

El rizo se evalúa en términos de curvatura de fibra, que describe la frecuencia de rizos que existe en la fibra (McGregor, 2002) o como el número de rizos por unidad de longitud (Hatcher y Atkins, 2000). Asimismo, se describe que la curvatura de las fibras presenta tres dimensiones, debido a que se encuentran flexionadas y torcidas a lo largo de su longitud. No obstante, debido a que la mayor parte de la curvatura ocurre en un plano y teniendo la flexión la mayor contribución, la forma de la fibra es representada en una onda bidimensional (Fish et al., 1999).

\section{Longitud de Fibra}

No se observaron diferencias significativas por sexo en la LM entre alpacas blancas y de color, donde el promedio varió entre 63 y $70 \mathrm{~mm}$. Empero, para dichos eventos es costumbre realizar recortes de las puntas del vellón a fin de presentar la mejor estampa del ejemplar. En consecuencia, dichos valores no representan el valor real de crecimiento de la fibra de alpaca, blanca o color. En cuanto 
a la categoría, la LM guarda alguna relación directa con la edad de la alpaca; pues animales de las categorías A1 exhiben las menores LM.

En cuanto a la categoría etaria, Siña (2012) refiere 110.5 y $87.8 \mathrm{~mm}$ para alpacas en Tacna, Vásquez et al. (2015) de 117.9 y 129.2 mm en Apurímac, Meza (2019) de 97.8 y 176.3 en Ayacucho y Quispe et al. (2020) de 102.2 y $79 \mathrm{~mm}$. Estos resultados reflejan una gran variabilidad en LM en función a factores ambientales y de edad. No obstante, en igualdad de condiciones, las fibras más largas producen hilos más fuertes, uniformes y delgados, y a su vez tienen mayor resistencia a la abrasión (Lamb, 2002). En sí, la LM depende de la frecuencia con que se esquilan los animales; y suele oscilar entre 40 y 150 mm (Bustinza, 2001).

\section{Correlación General}

Del conjunto de atributos analizados se infiere que i) el DM es la característica más importante por su influencia en el rendimiento del procesamiento y el valor de la fibra (Schlink, 2009; Botha y Hunter, 2010), ii) El FC, por su asociación aceptable con el DM $(\mathrm{r}=-0.840$ y $\mathrm{r}=-0.899)$ o regular con el DS(DM) y el FC y el IC, iii) Los altos valores del FC, por color, sexo y categoría, en especial de las alpacas blancas de los rebaños de plantel del Altiplano peruano ( $>95 \%)$ reflejan el nivel de presión selectiva aplicado en dichos rebaños y las bondades de comodidad adyacentes en el material genético de los CSD; iv) a través del apareamiento selectivo y sistemático es posible lograr el afinamiento de la fibra y la eliminación del factor picazón de la misma; además de alcanzar una mayor homogeneidad de la finura de la fibra en el vellón de la alpaca, blanca o color.

En suma, por sus características físicas, la fibra de alpaca es un producto versátil que influye en la comodidad del consumidor (Hatcher y Atkins, 2010; Swam, 2010), el rendimiento en el procesamiento, la durabilidad (McGregor y Postle, 2002) y los atributos textiles (Wood, 2003). Empero, la fibra de alpaca por su naturaleza biológica no es un producto uniforme, sino que sus atributos varían en función de factores ambientales y propios del animal.

\section{Correlaciones entre Atributos}

En el Cuadro 4 se muestra la matriz de los coeficientes de correlación de Pearson entre las principales características tecnológicas de fibra de alpacas Huacaya blanca y de color. Según, Martínez (1999), se puede considerar como asociaciones aceptables las halladas entre el DM y FC ( $r=-0.840 ; r=$ $0.899)$, asociación regular entre DM y $\mathrm{DS}(\mathrm{DM})(\mathrm{r}=0.669 ; \mathrm{r}=0.774), \mathrm{DS}(\mathrm{DM}) \mathrm{y}$ $\mathrm{FC}(\mathrm{r}=-0.777 ; \mathrm{r}=-0.745)$ y correlación mínima entre DM e IC $(r=-0.443 ; r=-0.521)$, $\mathrm{DS}(\mathrm{DM})$ e IC $(\mathrm{r}=-0.402 ; \mathrm{r}=-0.544)$, DS(DM) y CV(DM) $(r=0.562 ; r=0.498) ; y$ FC e IC $(r=0.442 ; r=0.511)$. Valores de coeficientes de correlación por debajo de $r=$ 0.30 indicarían ausencia de asociación.

Los resultados indican que, solo la mitad de las 15 correlaciones serian de interés explicativo y aplicativo; siendo las variables DM y FC las más importantes para la toma de decisiones en la industria textil, seguida de la DS(DM) y CV(DM).

\section{Conclusiones}

- Las distribuciones de frecuencias de los atributos de la fibra de las alpacas de plantel difieren de la curva normal, en distinto grado y según sea la variable. A excepción del FC, los atributos de la fibra presentan un sesgo de cola hacia la derecha; siendo moderada la asimetría del DM y ligera asimetría del IC. En tanto, las variables de dispersión, FC y LM presentan curvas muy asimétricas.

- Las características textiles de la fibra de alpacas blancas exhiben mayor finura que las de color $(\mathrm{p}<0.05)$, sin diferencia significativa para el factor sexo. 
- La DS(DM) y el CV(DM) de las fibras blancas, para ambos sexos, tienen menor dispersión $(\mathrm{p}<0.05)$

- El FC de las alpacas blancas presenta mayores valores que las de color $(p<0.05)$, sin diferencia significativa para el factor sexo.

- El IC de las alpacas blancas fue mejor que el de las alpacas de color $(\mathrm{p}<0.05)$.

- La mayoría de los atributos tiene relación con la edad de los animales, a excepción del CV(DM) que no muestra una tendencia clara.

- Los coeficientes de correlación de Pearson de las principales características tecnológicas de fibra se clasifican como asociación aceptable, regular y mínima.

\section{Literatura Citada}

1. Arango S. 2016. Variación del factor de confort en vellones de alpacas Huacaya con relación al sexo y edad. Tesis de Ingeniero Zootecnista. Lima Perú: Univ. Nacional Agraria La Molina. 56 p.

2. Atav R, Turkmen F. 2015. Investigation of the dyeing characteristics of alpaca fibers (Huacaya and Suri) in comparison with wool. Text Res J 85: 1331-1339. doi: 10.1177/0040517514563727

3. Aylan-Parker J, McGregor BA.2002. Optimising sampling techniques and estimating sampling variance of fleece quality attributes in alpacas. Small Ruminant Res 44: 53-64. doi: 10.1016/ S0921-4488(02)00038-X

4. Barrionuevo E. 2019. Caracterización física de la fibra de alpacas Huacaya utilizando OFDA 2000 en cuatro comunidades Ocongate, Quispicnachi. Tesis de Ingeniero Zootecnista. Cusco, Perú: Univ. Nacional San Antonio Abad. $84 \mathrm{p}$.

5. Botha AF, Hunter L. 2010. The measurement of wool fibre properties and their effect on worsted processing performance and product quality. Part 1:
The objective measurement of wool fibre properties. Textile Progress 42: 227-339. doi: 10.1080/00405167.2010.486932

6. Bustinza AV. 2001. La alpaca. Conocimiento del gran potencial andino. Puno, Perú: Univ. Nacional del Altiplano. 495 p.

7. Castillo R, Zacarías A. 2014. Determinación de las características tecnológicas de los diferentes componentes del vellón de la alpaca (Vicugna pacos) Huacaya. Tesis de Ingeniero Zootecnista. Perú: Univ. Nacional de Huancavelica. $130 \mathrm{p}$.

8. Contreras A. 2010. Caracterización de la fibra de alpacas Huacaya (Vicugna pacos) de color blanco. Tesis de Ingeniero Zootecnista. Perú: Univ. Nacional de Huancavelica. $83 \mathrm{p}$.

9. Cottle DJ. 2010. Australian sheep and wool. In: Cottle DJ (ed). International sheep and wool bandbook.UK: Nottingham University Press. p 49-72.

10. Cutiri R. 2019. Finura y medulación de la fibra de alpacas Huacaya de color blanco en las C.C. de Llullucha, Palcca y Accocunca Ocongate - Quispicanchi. Tesis de Ingeniero Zootecnista. Cusco, Perú: Univ. Nacional de San Antonio Abad. 80 p.

11. Fish VE, Mahar TJ, Crook BJ. 1999. Fibre curvature morphometry and measurement. Wool Technol Sheep Breeding 48: 248-265.

12. Gil R. 2017. Evaluación de las características textiles de la fibra de alpacas Huacaya del Instituto de Investigación y Promoción de Camélidos Sudamericanos, Puno. Tesis de Médico Veterinario y Zootecnista. Puno, Perú: Univ. Nacional del Altiplano. $67 \mathrm{p}$.

13. Hatcher S, Atkins KD. 2000. Breeding objectives which include fleece weight and fibre diameter do not need fibre curvature. Asian Austral J Anim 13: 293-296.

14. Holt CM. 2006. A survey of the relationships of crimp frequency, micron, character \& fibre curvature. In: The Australian Alpaca Association. 33 p. 
15. Lamb PR 2002. Metrology and processing research: implications for growers. Wool Tech Sheep Bree 50: 671-678

16. Machaca V, Bustinza AV, Corredor FA, Paucara V, Quispe EE, Machaca R. 2017. Características de la fibra de alpaca Huacaya de Cotaruse, Apurímac, Perú. Rev Inv Vet Peru 28: 843-851. doi: 10.15381/rivep.v28i4.13889.

17. Martínez C. 1999. Estadística y muestreo. Colombia: ECOE Ediciones. $886 \mathrm{p}$.

18. McColl A, Lupton C, Stobart B. 2004. Fiber characteristics of U.S. Huacaya alpaca. Alpaca Magazine 2004: 186-196.

19. McGregor BA, Postle R. 2002. Yarn knitted fabrics produced from low and high curvature superfine merino wool. Wool Tech Sheep Bree 50: 691-697.

20. McGregor BA. 2002. Comparative productivity and grazing behaviour of Huacaya alpacas and Peppin Merino sheep grazed on annual pastures. Small Ruminant Res 44: 219-232. doi: 10.1016/ S0921-4488(02)00050-0

21. McGregor BA, Butler K. 2004. Sources of variation in fibre diameter attributes of Australian alpacas and implications for fleece evaluation and animal. Aust J Agr Res 55: 43. doi: 10.1071/ AR03073

22. Meza M. 2018. Caracterización física de la fibra de alpacas de color de la raza Huacaya en el distrito de Totos, provincial Cangallo, region Ayacucho a 4438 msnm. Tesis de Médico Veterinario. Ayacucho, Perú: Univ. Nacional San Cristóbal de Huamanga. $60 \mathrm{p}$.

23. Quispe E. 2010. Evaluación de características productivas y textiles de la fibra de alpacas Huacaya de la región de Huancavelica, Perú. In: $33^{\text {th }}$ International Simposium on fiber South American Camelids. Huancavelica, Perú.
24. Quispe JE, Apaza E, Olarte C. 2020. Características físicas y perfil de diámetro de fibra de alpacas Huacaya del Centro Experimental La Raya (Puno, Perú), según edad y sexo. Rev Inv Vet Perú 32(2); e20004. doi: 10.15381/rivep.v3-2i2.20004

25. Quispe JE, Apaza E, Quispe DM, Morocco N. 2016. De vuelta a la alpaca: la producción primaria en una perspectiva empresarial y competitiva. Puno, Perú: Ed Meru. 440 p.

26. RGALLP. 2011. Reglamento de los registros genealógicos de alpacas y llamas del Perú. Aprobado por el DS No 0132011-AG. [Internet]. Disponible en: https:/ /www.minagri.gob.pe/portal/download/ pdf/marcolegal/normaslegales/decretossupremos/2011/ds13-2011-ag.pdf

27. Schlink T. 2009. Fibre diameter, staple strength, style, handle and curvature. In: WOOL412/513 Sheep Production - The Australian Wool Education Trust. $22 \mathrm{p}$.

28. Siña M. 2012. Características físicas de la fibra en alpacas Huacaya del distrito de Susapaya, provincia de Tarata. Tesis de Médico Veterinario Zootecnista. Tacna, Perú: Univ. Nacional Jorge Basadre Grohmann. 82 p.

29. Toma J, Rubio J. 2016. Estadística aplicada. Primera parte. $2^{\circ}$ ed. Lima, Perú: Univ. del Pacífico. $344 \mathrm{p}$.

30. Vásquez R, Gómez-Quispe O, Quispe E. 2015. Características tecnológicas de la fibra blanca de alpaca Huacaya en la zona altoandina de Apurímac. Rev Inv Vet Peru 26: 213-222. doi: 10.15381/ rivep.v26i2.11020.

31. Wang $X$, Wang L, Liu $X$. 2003. The quality and processing performance of alpaca fibres: a report for the Rural Industries Research and Development Corporation. Australia: RIRDC Publication $\mathrm{N}^{\mathrm{o}}$ 03/128. $132 \mathrm{p}$. 